\title{
不同亲疏水比例的光响应性嵌段共聚物的合成及溶液组装行为研究
}

\author{
丁妍春俞燕蕾韦嘉* \\ (复旦大学材料科学系 上海 200433)
}

\begin{abstract}
摘要 以末端带有二硫代酯基团的聚合物 $\mathrm{PhC}(=\mathrm{S})-\mathrm{S}-\mathrm{PEO}$ 作为大分子链转移剂, 利用可逆加成-断裂链转移自由基聚 合法(RAFT)合成一系列亲疏水比例不同的嵌段共聚物 $\mathrm{PEO}_{n}-b-\mathrm{PMeA} 6 \mathrm{AB} 2_{m}$, 并采用核磁共振 $\left({ }^{1} \mathrm{H} \mathrm{NMR}\right)$ 和凝胶渗透色 谱(GPC)对产物进行了表征. 运用场发射透射电子显微镜(FETEM)、扫描电子显微镜(SEM)、场发射扫描电子显微镜 (FESEM)、光学显微镜、粒径分析仪和荧光光谱研究了聚集体的形貌、粒径及其在光刺激下的响应行为. 结果表明，聚 集体形态为胶束, 包含大复合胶束. 随着自组装初始浓度的降低、转速的增加, 聚集体尺寸降低, 分布趋于均一; 当亲 疏水比例为 2.3 时, 球形复合胶束中出现了花生型、棒状结构. 包覆有尼罗红的聚集体在紫外/可见光的循环照射下, 部 分尼罗红被释放. 共聚物 BCP-S3-0.82 形成的自组装体系在紫外光照射下, 尺寸较大的复合胶束发生一定程度的不 可逆的解离.
\end{abstract}

关键词 可逆加成-断裂链转移自由基聚合法; 两亲性嵌段共聚物; 偶氮苯基团; 亲疏水比例; 自组装; 胶束

\section{Synthesis and Self-assembly Behavior of Photo-responsive Diblock Copolymers with Different Hydrophilic/Hydrophobic Ratios}

\author{
Ding, Yanchun Yu, Yanlei Wei, Jia* \\ (Department of Materials Science, Fudan University, Shanghai 200433)
}

\begin{abstract}
A series of diblock copolymers, poly(ethylene oxide)-block-poly\{6-[4-(4-ethoxyphenylazo) phenoxy] hexyl methacrylate (PEO-b-PMA6AB2), with different hydrophilic/hydrophobic ratios were synthesized by reversible addition-fragmentation chain transfer (RAFT) polymerization. The structures and properties of diblock copolymers were characterized by ${ }^{1} \mathrm{H}$ NMR and gel permeation chromatography (GPC). The self-assembly method was as follows: block copolymers were dissolved in tetrahydrofuran (THF) at first, and then by adding water at the rate of $2.5 \mu \mathrm{L} / \mathrm{s}$, the aggregates self-assembled when the critical micelle concentration (CMC) was reached. Field emission transmission electron microscope (FETEM), scanning electron microscope (SEM), field emission scanning electron microscope (FESEM), optical microscope, particle size analyzer, UV-Vis absorption spectroscope and fluorescence emission spectroscope were used to study the morphology and size of the aggregates and their stimuli-responsive behavior. The SEM and FETEM results showed that polymeric micelles and large compound micelles (LCM) coexisted in the aqueous solution. Furthermore, it was found that the size of the self-assembly reduced with the decrease of initial concentration and the increase of stirring rate, and the distribution tended to be uniform. Meanwhile, the morphology of large compound micelles turned to be different, changing from common sphere to peanut shape or rod-like shape when the hydrophilic/hydrophobic ratio was 2.3. By encapsulating Nile Red into the aggregates, the peanut shape and rod-like shape were captured by fluorescence microscope. UV-Vis absorption spectra of the self-assembly revealed that the photo-responsive behavior of azobenzene moieties in the aggregates was reversible but restricted to some extent. The change on fluorescence intensity of Nile Red encapsulated in the diblock copolymer aggregates indicated that the alternative irradiation of UV and visible light induced the release of Nile Red resulted from the photoisomerization of the azobenzene moieties. In addition, the photo-responsive study showed that large compound micelles self-assembled from BCP-S3-0.82 could dissociate under the irradiation of UV light. Other large compound micelles assembled from the rest of block copolymers also dissociated, but the extent of the dissociation was not as remarkable as BCP-S3-0.82, which had the maximal hydrophobic ratio.
\end{abstract}

Keywords RAFT; amphiphilic block copolymer; azobenzene moiety; hydrophilic/hydrophobic ratio; self-assembly; micelles

\footnotetext{
*E-mail: weijia@fudan.edu.cn

Received January 23, 2014; published March 29, 2014.

Supporting information for this article is available free of charge via the Internet at http://sioc-journal.cn.

Project supported by the National Natural Science Foundation of China (Nos. 21134003, 21273048, 51225304, 51203023), Fundamental Research Funds for the Central Universities (No. 20520133189) and Shanghai Natural Science Foundation (12ZR1401600).

项目受国家自然科学基金(Nos. 21134003, 21273048, 51225304, 51203023)、复旦大学中央高校基本科研业务费青年教师科研能力提升项目(No. 20520133189)和上海市自然科学基金(No. 12ZR1401600)资助.
} 


\section{1 引言}

两亲性嵌段共聚物是由两种或两种以上不同结构 的分子链段通过化学键结合在一起的聚合物. 在能量最 低原理驱动下, 该类聚合物在选择性溶剂中, 能自组装 形成不同形态的聚集体, 如胶束、囊泡等. 目前大量的 研究工作将该聚集体应用于纳米-微米级反应器、催化 剂载体、介孔材料的模板以及药物释放载体等领域 ${ }^{[1 \sim 4]}$.

在光响应性材料领域中, 主/侧链含有偶氮类生色 团的聚合物得到了广泛地研究 ${ }^{[2]}$. 偶氮苯官能团能在紫 外光/可见光的交替作用下, 发生可逆的光致顺反异构 化反应 ${ }^{[5]}$. 偶氮苯的反式(trans) 和顺式( cis) 异构体在分 子尺寸、极性上有较大区别. 在紫外光的作用下, 处于 低能态的 trans 体中氮一氮双键产生电子跃迁, 引起键的 旋转, 形成 $c i s$ 体. 而 $c i s$ 体能够在可见光照射或者热松 弛作用下回复到 trans 体. 若在两亲性嵌段共聚物中引 入偶氮苯基团, 则形成的聚集体可实现光致解离 ${ }^{[6]}$ 、光 致融合 ${ }^{[7]}$ 、光致变形 ${ }^{[8]}$ 等响应性行为.

各种新型的活性聚合体系已应用到嵌段共聚物的 合成中, 其中最常用的方法为原子转移自由基聚合 (ATRP) 和可逆加成一断裂链转移聚合(RAFT), 两者各有 优缺点. 与 ATRP 相比, RAFT 聚合适用的单体更广, 有 利于具有特殊官能团的烯类单体的聚合, 同时避免了使 用过渡金属类催化剂. 本课题组曾用 ATRP 聚合方法制 备了含偶氮类的嵌段共聚物 ${ }^{[9 \sim 11]}$. 由于使用的偶氮烯类 单体活性较低, 所以实验过程中需使用高活性的配体 (Me6TREN)作为催化剂, 该配体制备过程较为繁琐. 故 本文报道了以RAFT活性聚合制备含偶氮苯的两亲性嵌 段共聚物的方法. 该方法以末端带有二硫代酯基团的聚 合物 $\mathrm{PhC}(=\mathrm{S})-\mathrm{S}-\mathrm{PEO}$ 为大分子链转移剂, 有效简化实 验操作, 提高聚合成功率. 在此基础上, 利用共溶剂沉 淀法制备了具有光响应性的聚集体, 并讨论了初始浓 度、搅拌速度及亲疏水比例对聚集体形态的影响, 研究 了由不同亲疏水比例的嵌段共聚物形成的聚集体的光 响应性行为.

\section{2 结果与讨论}

\section{1 聚合物结构表征}

图 1 为该嵌段共聚物 PEO- $b$-PMeA6AB2 典型的 ${ }^{1} \mathrm{H}$ NMR 图谱.

$\delta 6.9 \sim 7.0$ (峰 $\mathrm{c}$ )和 $\delta 7.8 \sim 7.9$ (峰 $\mathrm{d}$ )的振动峰对应于 偶氮苯基团上 $\mathrm{H}$ 原子的特征峰; $\delta 3.3 \sim 3.4$ (峰 a)之间的 质子峰对应于 $\mathrm{PEO}$ 端基- $\mathrm{CH}_{3}$ 上的 $\mathrm{H}$ 原子; $\delta 3.5 \sim 3.7$ (峰 b)之间的质子峰对应于 $\mathrm{PEO}$ 嵌段- $\mathrm{CH}_{2} \mathrm{CH}_{2} \mathrm{O}$ 一上的氢. 根据核磁上对应特征峰的积分面积, 同时结合 GPC 测 得的分子量, 得到不同亲疏水比例的嵌段共聚物的组 成, 如表 1 所示. BCP-L1-3.6, BCP-L2-2.5 表示分子量为 $5000 \mathrm{~g} / \mathrm{mol}$ 的 PEO 作为亲水嵌段, 亲疏水比例分别为

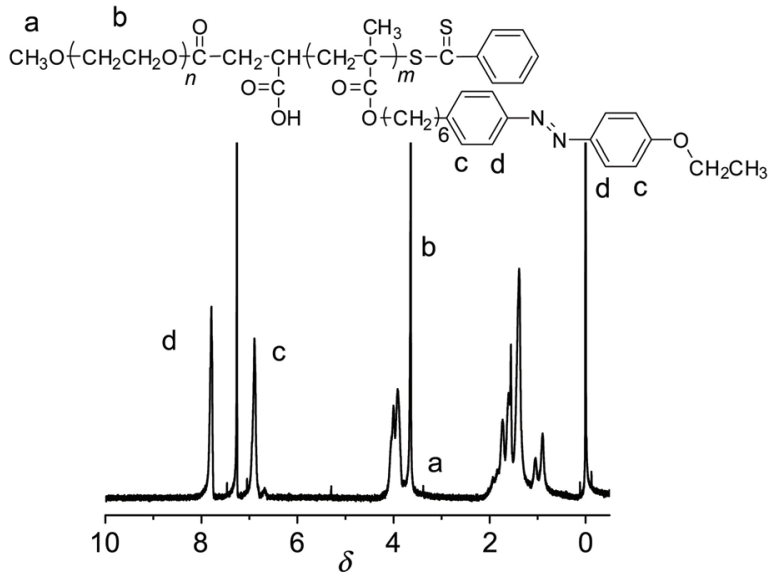

图 1 PEO- $b$-PMeA6AB2 的 ${ }^{1} \mathrm{H}$ NMR 谱图

Figure $1{ }^{1} \mathrm{H}$ NMR spectrum of PEO- $b$-PMeA6AB2

表 1 具有不同亲疏水比例的两亲性嵌段共聚物的结构表征

Table 1 Structure characterization of amphiphilic copolymers with different hydrophilic/hydrophobic ratios

\begin{tabular}{|c|c|c|c|}
\hline 样品 & 组成 & $M_{\mathrm{n}}(\mathrm{g} / \mathrm{mol})$ & PDI \\
\hline BCP-L $1^{a}-3.6^{b}$ & $\mathrm{PEO}_{128}-b-\mathrm{PMeA} 6 \mathrm{AB} 2_{36}$ & $1.5 \times 10^{4}$ & 1.57 \\
\hline BCP-L2-2.5 & $\mathrm{PEO}_{128}-b-\mathrm{PMeA} 6 \mathrm{AB} 2_{51}$ & $2.2 \times 10^{4}$ & 1.39 \\
\hline $\mathrm{BCP}-\mathrm{S} 1^{c}-2.3$ & $\mathrm{PEO}_{50}-b-\mathrm{PMeA} 6 \mathrm{AB} 2_{22}$ & $1.1 \times 10^{4}$ & 1.86 \\
\hline BCP-S2-1.4 & $\mathrm{PEO}_{50}-b-\mathrm{PMeA} 6 \mathrm{AB} 2_{37}$ & $1.2 \times 10^{4}$ & 1.53 \\
\hline BCP-S3-0.82 & $\mathrm{PEO}_{50}-b-\mathrm{PMeA} 6 \mathrm{AB} 2_{61}$ & $2.3 \times 10^{4}$ & 1.46 \\
\hline
\end{tabular}

${ }^{a} \mathrm{~L}$ 表示 PEO 的分子量为 $5000 ;{ }^{b}$ 亲疏水重复单元的摩尔比例; ${ }^{c} \mathrm{~S}$ 表示 $\mathrm{PEO}$ 的分子量为 2000 .

3.6 和 2.5 的嵌段共聚物. BCP-S1-2.3, BCP-S2-1.4, BCP-S3-0.82 中的 PEO 链段较短, 其分子量为 2000 $\mathrm{g} / \mathrm{mol}$, 亲疏水比例是 $2.3,1.4$ 以及 0.82 .

\section{2 自组装行为研究}

\subsection{1 组装条件对聚集体的影响}

在选择性溶剂中, 两亲性嵌段共聚物某一嵌段不能 溶解在其中而趋于聚集, 另一嵌段具有较好的溶解性则 阻止了沉淀的生成. PEO 为典型的亲水性链段, $\mathrm{PMeA} 6 \mathrm{AB} 2$ 为疏水部分, 故 $\mathrm{PEO}_{n}-b-\mathrm{PMeA} 6 \mathrm{AB} 2_{m}$ 在水 中可发生自组装.

采用扫描电子显微镜(SEM)及场发射透射电子显微 镜 (FETEM) 确定聚集体的形态. 图 2(a) 2(c) 为 BCP-L1-3.6 聚集体的 SEM 图像，表征聚集体形貌：该 组装体中存在大量纳米级的小球及相对较少的近微米 级的大球. 图 2(d)为聚集体的 FETEM 图像, 确定聚集 体形态为胶束: 纳米级的实心小球说明该聚集体中存在 球形的胶束; 而直径较大的实心球则为大复合胶束 (large compound micelles, LCM $)^{[12]}$; 故自组装体系中以 纳米级胶束为主体，同时存在微纳米级的大复合胶束， 其他嵌段共聚物的组装体形态与上述一致. 大复合胶束 为一个多核聚集体，当嵌段共聚物中的疏水段比例较高 时，易组装形成. 其由反相胶束构成，这些反相胶束的 
<smiles>C=C(C)C(=O)OCCOc1ccc(N=Nc2ccc(OCC)cc2)cc1</smiles>

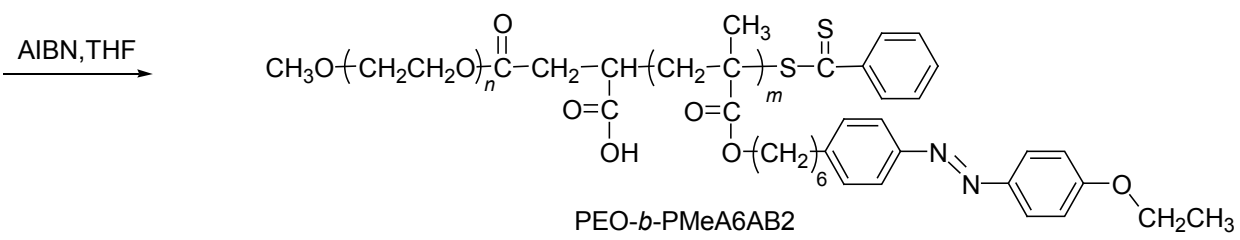

图式 1 两嵌段共聚物 PEO- $b$-PMeA6AB2 的合成路线

Scheme 1 The synthetic route of PEO- $b$-PMeA6AB2
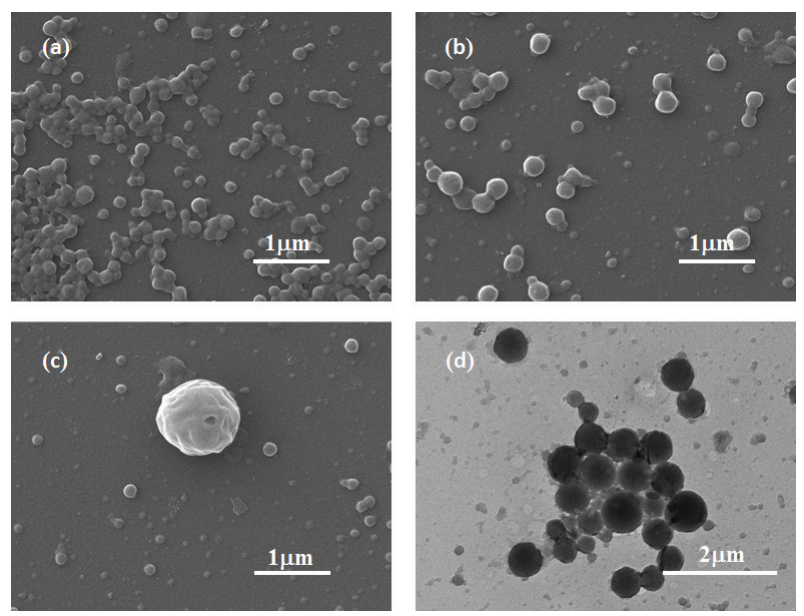

图 2 BCP-L1-3.6 聚集体的 SEM 图像(a), (b), (c)和 FETEM 图像(d) Figure 2 SEM images (a), (b), (c) and FETEM image (d) of aggregates self-assembled from BCP-L1-3.6

核为 PEO, 壳为 PMeA6AB2.

通过对聚集体的粒径测试来研究组装条件, 如初始 浓度、搅拌速度对聚集体的尺寸及分布的影响.

从图 3(a)中可以看出, 当转速固定为 $2000 \mathrm{r} / \mathrm{min}$ 时, 随着初始浓度降低, 聚集体(含胶束及大复合胶束)尺寸 变小, 分布变窄. 初始浓度从 $2 \mathrm{mg} / 200 \mu \mathrm{L}$ 降低到 1 $\mathrm{mg} / 200 \mu \mathrm{L} 、 0.5 \mathrm{mg} / 200 \mu \mathrm{L}$ 时, 自组装形成的聚集体平 均直径从 $327 \mathrm{~nm}$ 依次降低到 297, $260 \mathrm{~nm}$, 尺寸分布随 之趋于集中, PDI 从 0.437 降到 $0.263,0.140$. 在同样的组 装条件下, 初始浓度增加, 使更多的分子链进入同一聚 集体, 因此导致形成的聚集体尺寸变大 ${ }^{[13]}$.

根据图 3(b)可得到转速对聚集体尺寸的影响. 当初 始浓度为 $3 \mathrm{mg} / 200 \mu \mathrm{L}$, 随着转速从 $200 \mathrm{r} / \mathrm{min}$ 增加到 $2000 \mathrm{r} / \mathrm{min}$, 聚集体的直径从 $409 \mathrm{~nm}$ 减小到 $319 \mathrm{~nm}$, 同 时分散性趋于均一, PDI 从 0.429 降到 0.209. 转速增加 导致自组装过程中受到的剪切力增加, 有效减小了聚集 体的尺寸，使得分布均一化. (a)

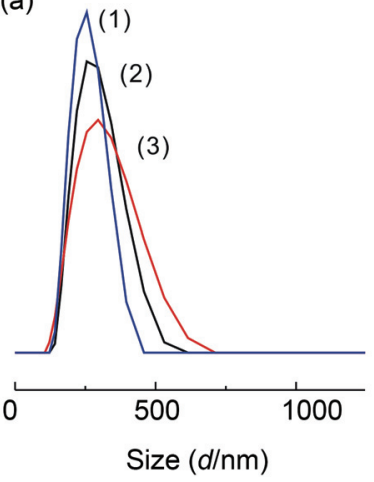

(b)

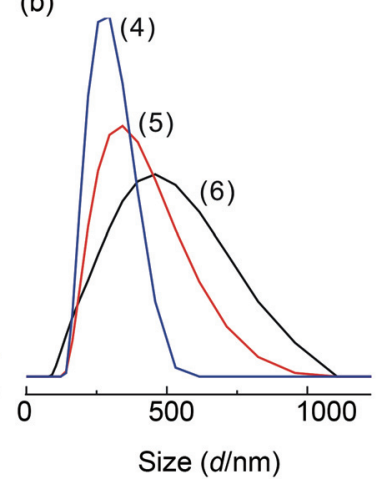

图 3 (a)转速为 $2000 \mathrm{r} / \mathrm{min}$, 不同初始浓度 $0.5 \mathrm{mg} / 200 \mu \mathrm{L}$ (1), 1 $\mathrm{mg} / 200 \mu \mathrm{L}(2), 2 \mathrm{mg} / 200 \mu \mathrm{L}$ (3)及(b)初始浓度为 $3 \mathrm{mg} / 200 \mu \mathrm{L}$, 不同转 速 $2000 \mathrm{r} / \mathrm{min}$ (4), $1400 \mathrm{r} / \mathrm{min}$ (5), $200 \mathrm{r} / \mathrm{min}$ (6)下制备得到的 BCP-L1-3.6 聚集体水溶液直径分布

Figure 3 Size distribution of the BCP-L1-3.6 aggregates with (a) different initial concentration of $0.5 \mathrm{mg} / 200 \mu \mathrm{L}(1), 1 \mathrm{mg} / 200 \mu \mathrm{L}$ (2), 2 $\mathrm{mg} / 200 \mu \mathrm{L}$ (3) under the stirring rates of $2000 \mathrm{r} / \mathrm{min}$; (b) different stirring rate of $2000 \mathrm{r} / \mathrm{min}(4), 1400 \mathrm{r} / \mathrm{min}(5), 200 \mathrm{r} / \mathrm{min}$ (6) under the initial concentration of $3 \mathrm{mg} / 200 \mu \mathrm{L}$

\subsection{2 亲疏水比例对自组装形态影响}

在相同的组装条件下(初始浓度为 $2 \mathrm{mg} / 200 \mu \mathrm{L}$, 转 速 $800 \mathrm{r} / \mathrm{min}$ ), 不同亲疏水比例的嵌段共聚物形成的聚 集体形貌发生变化.

通过场发射透射电子显微镜(FETEM)对大复合胶 束进一步分析, 发现 BCP-S1-2.3 具有特殊的聚集体结 构. 图 4(a), 4(b), 4(d), 4(e)中复合胶束形貌均为球形; 具 有最高疏水比例的嵌段共聚物 BCP-S3-0.82 形成的复合 胶束边缘有一定的变形, 这是由于其内部溶剂含量较高 导致. 而 4(c)图表明, 当亲疏水比例为 2.3 时, 复合胶束 形貌发生了一定变化. 改变亲疏水比例, 组装过程受热 力学影响, 形貌发生了变化, 出现花生型、棒状大复合 胶束. 利用场发射透射电子显微镜(FETEM), 场发射扫 描电子显微镜(FESEM)及苂光显微镜均观察到了上述 特殊结构, 分别见图 5(a), 5(b), 5(c), 而图 5(d) 为对照实 

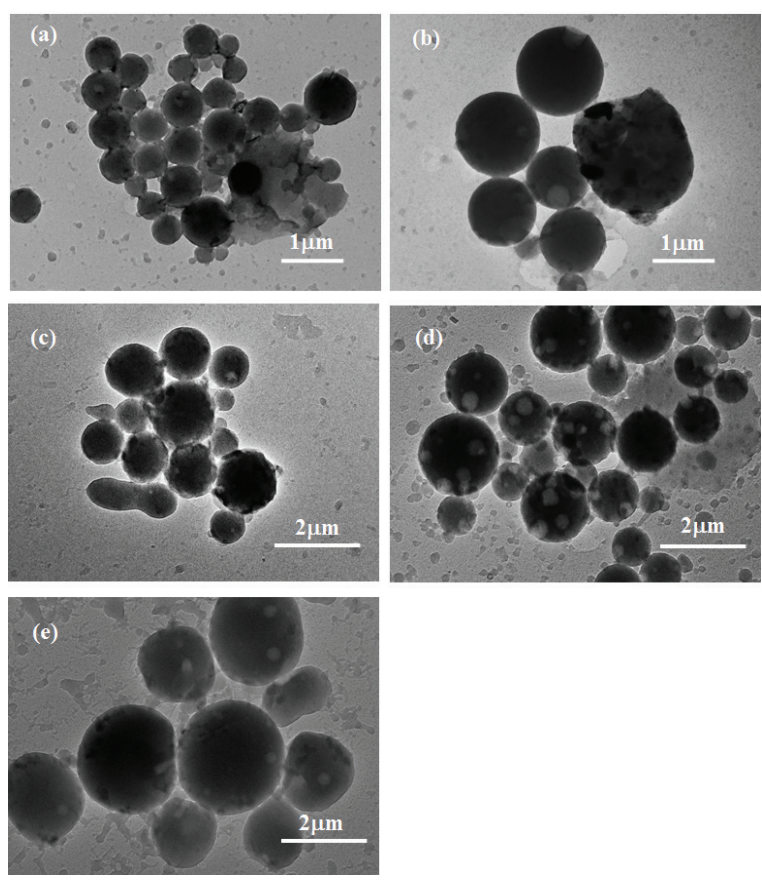

图 4 亲疏水比例不同的嵌段共聚物形成聚集体的 FETEM 图像 BCP-L1-3.6 (a), BCP-L2-2.5 (b), BCP-S1-2.3 (c), BCP-S2-1.4 (d) BCP-S3-0.82 (e)

Figure 4 FETEM images of self-assembled aggregates with different hydrophilic/hydrophobic ratios BCP-L1-3.6 (a), BCP-L2-2.5 (b), BCP-S1-2.3 (c), BCP-S2-1.4(d), BCP-S3-0.82 (e)
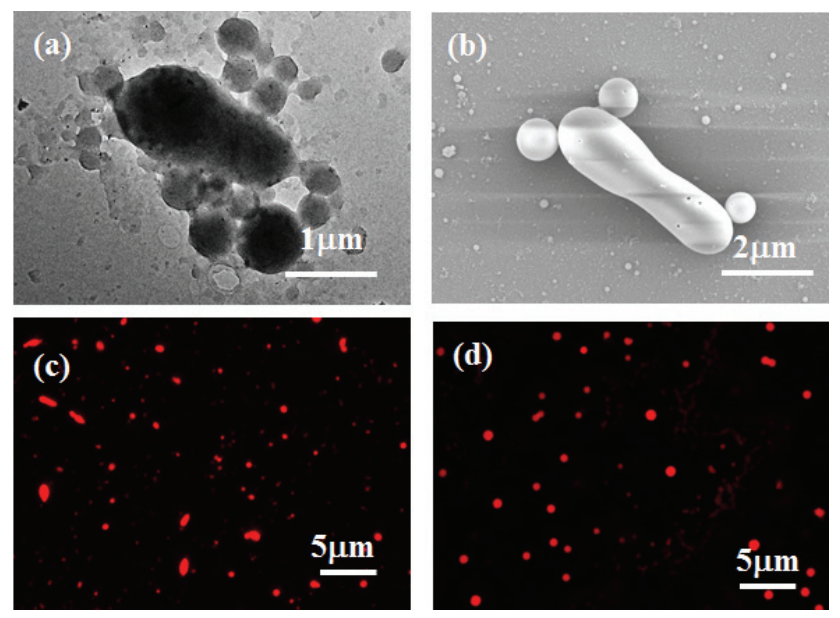

图 5 BCP-S1-2.3 聚集体的 FETEM 图像(a), FESEM 图像(b), 以及 BCP-S1-2.3 (c), BCP-L1-3.6 (d)聚集体中包覆有尼罗红的苂光显微镜 照片

Figure 5 FETEM (a) and FESEM (b) image of aggregates self-assembled from BCP-S1-2.3, and fluorescence microscope images of BCP-S1-2.3 (c) and BCP-L1-3.6 (d) aggregates encapsulated with Nile Red

验的结果, 是包覆有尼罗红的 BCP-L1-3.6 聚集体的荧 光照片. 此外, 在电镜样品制备过程中, 由于大复合胶 束内部含有较多的溶剂, 在干燥过程中, 不同区域溶剂 挥发速度不同, 会导致干燥之后的样品表面观测到一些 小孔结构, 如图 S1 所示.
结合粒径测试结果(表 2)及多种显微镜图像(图 4 和 图 5), 说明不同亲疏水比例的嵌段共聚物形成的聚集体 中包含纳米级的胶束及微纳米级的复合胶束, 并以胶束 为主. 同时, 当亲疏水比例为 2.3 时, 形成了花生型、棒 状结构. 相关研究曾表明 ${ }^{[14]}$, 引起自组装体形貌改变的 因素包括共聚物亲疏水链段长度比、溶剂组成、温度以 及添加剂等. 当其中的一个参数改变时, 比如亲疏水比 例发生变化, 形成聚集体结构的平衡会被打破, 导致聚 集体形貌转变 ${ }^{[15]}$.

\section{3 光响应性研究}

\subsection{1 聚集体光致异构化行为的研究}

通过紫外可见吸收光谱, 我们研究了聚集体的光响 应性. 图 S2 为 PEO- $b$-PMeA6AB2 组装形成的聚集体在 光照下的紫外可见吸收光谱图. 图 S2(a)表示聚集体水 溶液在紫外光每间隔 $5 \mathrm{~s}$ 照射下, 波长 $365 \mathrm{~nm}$ 处 (trans 偶氮苯 $\pi-\pi *$ 吸收)的峰强逐渐减弱, $460 \mathrm{~nm}$ 处( $i s$ 偶氮苯 $\mathrm{n}-\pi^{*}$ 吸收)的峰强逐渐增强. 而图 S2(b)表示经过紫外光 照射达到光平衡的聚集体水溶液在可见光照射下, $c i s$ 体 偶氮苯含量逐渐增加, trans 偶氮苯含量逐渐减少. 该结 果说明偶氮苯基团虽然包覆在聚集体中，但是其光致异 构化过程仍然可逆.

尼罗红是一种常用的疏水荧光染料, 几乎不溶于 水, 在水环境中它的荧光非常弱 ${ }^{[15 ~ 17]}$. 当尼罗红被包覆 在胶束的疏水部分中时, 尼罗红能发出强荧光. 若尼罗 红所处环境的极性增加则会导致尼罗红荧光猝灭.

图 6 表示包覆有尼罗红的胶束水溶液在紫外/可见 光照射后的苂光发射光谱. 从图 6(a)中可知, 随着紫外 光照射时间增加, 尼罗红苂光强度逐渐减弱. 图 6(b)为 经过紫外光照射达到光平衡之后的聚集体水溶液在可 见光照射不同时间后，尼罗红的荧光发射强度逐渐增 强. 根据对照实验, 尼罗红的荧光强度不随光照变化, 而该共聚物形成的聚集体水溶液并不存在荧光发射(见 图 S3). 所以, 紫外/可见光引起的尼罗红荧光强度的变 化是由于偶氮苯基团的光响应性引起的. 紫外光照射 下, 偶氮苯 trans 体转变成 $c i s$ 体, 尼罗红所处的微环境 极性增强. 这在很大程度上导致尼罗红的苂光强度减 弱. 而根据图 6(c)和(d)可以看出, 在紫外光及可见光的 循环照射之后，最大荧光发射值逐渐降低，这说明在尼 罗红有一部分释放到水环境中. 因为棒状的 trans 体转 变成弯曲的 $c i s$ 体后，聚集体内部的有序堆积程度下降， 同时紫外光照射下大复合胶束存在一定程度的解离, 故 有可能导致一部分尼罗红向外释放.

\subsection{2 聚集体形貌光响应性的研究}

对嵌段共聚物 $\mathrm{PEO}_{n}-b-\mathrm{PMeA} 6 \mathrm{AB} 2_{m}$ 进行了聚集体 形貌光响应性的相关研究, 结果表明聚集体中大复合胶 束随着紫外光的照射出现不可逆的解离, 形貌的变化跟 偶氮含量直接相关. 偶氮含量越高, 其解离程度越明显. 

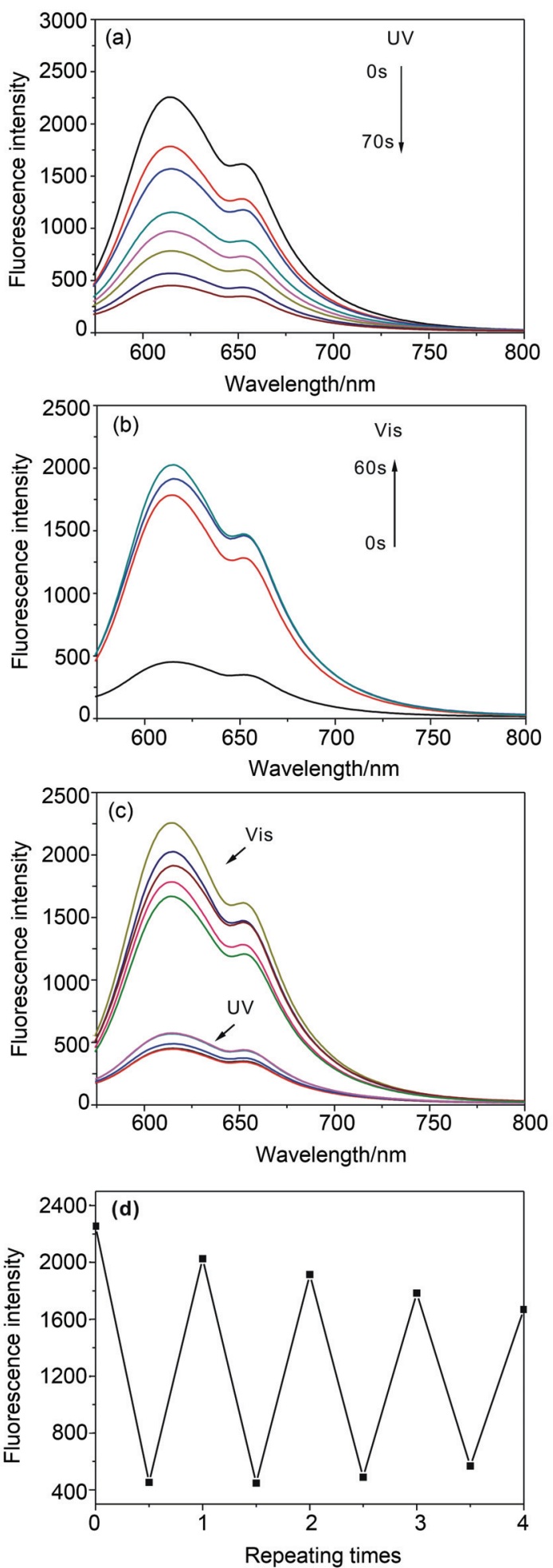

图 6 包覆有尼罗红的 BCP-S3-0.82 聚集体水溶液 $(0.4 \mathrm{mg} / \mathrm{mL})$ 在紫外 光 $\left(365 \mathrm{~nm}, 90 \mathrm{~mW} / \mathrm{cm}^{2}\right)(\mathrm{a})$ 和可见光 $\left(530 \mathrm{~nm}, 50 \mathrm{~mW} / \mathrm{cm}^{2}\right)(\mathrm{b})$ 照射不同 时间后的苂光发射光谱; 经过紫外-可见光循环照射后的荧光发射光 谱(c)和对应荧光强度的峰值(d)

Figure 6 Fluorescence spectra of $0.4 \mathrm{mg} / \mathrm{mL}$ aqueous solution of polymeric aggregates encapsulated with Nile Red under the irradiation of UV light (365 nm, $\left.90 \mathrm{~mW} / \mathrm{cm}^{2}\right)$ (a), visible light $\left(530 \mathrm{~nm}, 50 \mathrm{~mW} / \mathrm{cm}^{2}\right)$ (b), and alternating UV and visible light irradiation to the photostationary state (c); (d) shows maximum fluorescence intensity related to (c)
图 7 为具有较高偶氮苯比例的 BCP-S3-0.82 在紫外光/ 可见光照射下的高分辨率光学显微镜图像.
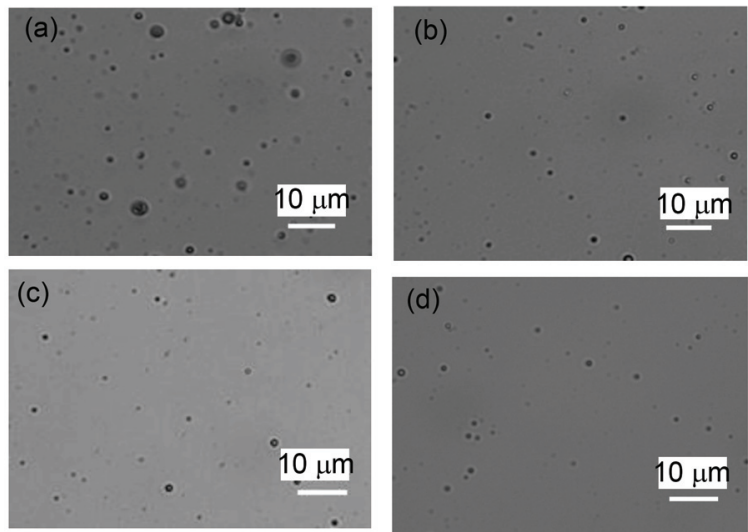

图 7 BCP-S3-0.82 形成的聚集体未光照射之前(a)以及紫外光 $(365 \mathrm{~nm}$, $150 \mathrm{~mW} / \mathrm{cm}^{2}$ )照射 $5 \mathrm{~min}(\mathrm{~b})$, 紫外光 $\left(365 \mathrm{~nm}, 150 \mathrm{~mW} / \mathrm{cm}^{2}\right)$ 照射 10 $\min (\mathrm{c})$, 可见光 $\left(530 \mathrm{~nm}, 50 \mathrm{~mW} / \mathrm{cm}^{2}\right.$ )照射 $20 \mathrm{~min}(\mathrm{~d})$ 后的形貌

Figure7 Morphology of aggregates self-assembled from BCP-S3-0.82 before UV irradiation (a) and after UV $\left(365 \mathrm{~nm}, 150 \mathrm{~mW} / \mathrm{cm}^{2}\right)$ irradiation for $5 \mathrm{~min}$ (b), for $10 \mathrm{~min}$ (c) and after visible light $\left(530 \mathrm{~nm}, 50 \mathrm{~mW} / \mathrm{cm}^{2}\right)$ irradiation for $20 \mathrm{~min}(\mathrm{~d})$

在搅拌条件下, BCP-S3-0.82 聚集体在紫外光(365 $\left.\mathrm{nm}, 150 \mathrm{~mW} / \mathrm{cm}^{2}\right)$ 的照射下，尺寸较大的复合胶束会发 生解离; 在可见光 $\left(530 \mathrm{~nm}, 50 \mathrm{~mW} / \mathrm{cm}^{2}\right)$ 的照射下, 解离 的复合胶束不能够回复. 这种形貌的变化, 主要是由于 偶氮苯从 trans 体转变成 cis 体之后, 疏水链段在水溶 液中的溶解性增强造成的 ${ }^{[18]}$.

\section{3 结论}

本文采用了 RAFT 聚合方法制备了一系列不同分子 量的两亲性嵌段共聚物 $\mathrm{PEO}_{n}-b-\mathrm{PMeA} 6 \mathrm{AB} 2_{m}$. 通过共溶 剂沉淀法形成的聚集体为胶束, 包含大复合胶束. 聚集 体尺寸受制备条件的影响, 随着初始浓度的降低、转速 的增大，尺寸变小且趋于均一. 聚集体的形貌随着亲疏 水比例的改变而发生变化. 当亲疏水比例为 2.3 时, 球 形复合胶束中出现花生型以及棒状结构. 共聚物组装成 胶束后，虽然偶氮苯基团受到一定空间位阻的限制，但 其异构化过程仍可逆. 而包覆有尼罗红的聚集体水溶液 在紫外光/可见光的循环照射之后, 有一部分尼罗红释 放到水环境中. 具有较高偶氮苯比例的 BCP-S3-0.82 形 成的自组装体在紫外光照射下，聚集体中尺寸较大的复 合胶束会发生解离; 在可见光的照射下，解离不可回复.

\section{4 实验部分}

\section{1 原料}

4-溴苯(分析纯), 二硫化碳(分析纯), 四氯化碳(分 析纯), 乙醚(分析纯), 甲苯(分析纯), 四氢呋喃(分析 纯), $N, N$-二甲基甲酰胺(分析纯), 均购于上海凌峰化学 
试剂有限公司; 偶氮二异丁腈(分析纯), 马来酸䣶(分析 纯), 镁条(分析纯)均购于国药集团化学试剂有限公司.

6-氯-1-已醇 $(97 \%), 4$-乙氧基苯胺( $98 \%)$, 甲基丙烯 酰氯, 聚乙二醇 $\left(M_{\mathrm{n}}=2000 \mathrm{~g} / \mathrm{mol}\right.$ 或 $\left.5000 \mathrm{~g} / \mathrm{mol}\right)$ 均购于 Alfa Aesar.

药品纯化说明: 四氢呋喃乙醚通过加金属钠进行蒸 馏纯化(二苯甲酮为指示剂), 偶氮二异丁腈用乙醇重结 晶, 镁条使用前用砂纸处理氧化层, 四氯化碳、溴苯和 甲苯使用前均用无水硫酸镁干燥处理.

\section{2 二硫代苯甲酸(DTBA)的合成}

二硫代苯甲酸的合成基于文献 ${ }^{[19]}$ 所述方法. 在 250 $\mathrm{mL}$ 的三颈瓶中加入镁屑 $(1 \mathrm{~g}, 41.7 \mathrm{mmol})$ 、无水乙醚 $(20$ $\mathrm{mL})$, 在滴液漏斗中加入乙醚 $(40 \mathrm{~mL})$ 和溴苯 $(4.4 \mathrm{~mL}$, $41.7 \mathrm{~mL})$, 反应 $1 \mathrm{~h}$ 得到格氏试剂. 将制备得到的格氏试 剂以及二硫化碳 $(2.5 \mathrm{~mL}) 、 \mathrm{THF}(30 \mathrm{~mL})$ 加入到 $250 \mathrm{~mL}$ 希丁克瓶中, 低温 $\left(-5{ }^{\circ} \mathrm{C}\right)$ 下反应 $1 \mathrm{~h}$. 然后, 在反应体 系中加入 $10 \%$ 的冰盐酸 $(60 \mathrm{~mL})$ 酸化, 并用乙醚萃取, 分 出深红色有几层. 有机层用 $10 \%$ 的冷 $\mathrm{NaOH}(100 \mathrm{~mL})$ 溶 液处理. 得到的碱液用乙醚洗涤三次, 然后用冷的盐酸 酸化处理, 直至水相呈无色. 最后用无水乙醚萃取, 旋 转蒸发得到 $6.4 \mathrm{~g}$ 红色油状的二硫代苯甲酸.

\section{3 大分子引发剂 PEO-S 的合成}

分子量 $2000 \mathrm{~g} / \mathrm{mol}$ 的 PEO-OH (3 g, $1.5 \mathrm{mmol})$ 或者 分子量 $5000 \mathrm{~g} / \mathrm{mol}$ 的 PEO-OH (7.5 g, $1.5 \mathrm{mmol})$ 溶于 40 $\mathrm{mL}$ 甲苯中, 加入马来酸酤 $(1 \mathrm{~g}, 10 \mathrm{mmol})$, 在 $250 \mathrm{~mL}$ 的 希丁克瓶中 $60{ }^{\circ} \mathrm{C}$ 搅拌反应 $24 \mathrm{~h}$. 采用旋转蒸发的方式 除去反应液中的大部分甲苯, 并溶于二氯甲烷中, 在大 量乙醚中沉淀除去未反应的马来酸酐, 过滤, 烘干得到 $2.5 \mathrm{~g}$ 的 PEO2000-MAh 以及 $6.2 \mathrm{~g}$ 的 PEO5000-MAh. 将 反应得到的 PEO2000-MAh (2 g, $1 \mathrm{mmol})$ 或者 PEO5000MAh (5 g, $1 \mathrm{mmol})$ 和二硫代苯甲酸 $(0.3 \mathrm{~g}, 13 \mathrm{mmol})$ 加入 到四氯化碳 $(40 \mathrm{~mL})$ 中, 在 $65{ }^{\circ} \mathrm{C}$ 下搅拌反应 $24 \mathrm{~h}$. 初产 物在大量乙醚中沉淀 3 次, 除去未反应完的 DTBA. 烘 干得到大分子引发剂 $\mathrm{PhC}(=\mathrm{S})-\mathrm{S}-\mathrm{PEO}$ (PEO-S), PEO2000-S 0.9 g, PEO5000-S $1.1 \mathrm{~g}$.

\section{4 两亲性嵌段共聚物 $\mathrm{PEO}_{n}-b-\mathrm{PMeA6AB2} 2_{m}$ 的合成}

在 $25 \mathrm{~mL}$ 的希丁克瓶中, 加入 AIBN (2 mg, 0.01 $\mathrm{mmol})$, 偶氮苯单体 MeA6AB2 (0.3 g, $0.7 \mathrm{mmol})$, 大分 子引发剂 PEO-S (0.2 g). 用氩气充满希丁克瓶, 加入 THF $2 \mathrm{~mL}$. 用液氮将反应体系冷冻, 抽真空, 接着再解 冻, 实现冷冻-抽真空-解冻循环 3 次. 将希丁克瓶置于 $70{ }^{\circ} \mathrm{C}$ 油浴中搅拌反应 $48 \mathrm{~h}$. 反应结束后, 将初产物旋 转蒸发, 溶于少量二氯甲烷中, 在大量乙醚中沉淀出去 未反应的偶氮单体以及 AIBN, 干燥, 得到嵌段共聚物.

\section{5 胶束的制备}

采用文献 ${ }^{[20]}$ 中介绍的方法: 共溶剂沉淀法制备嵌
段共聚物的自组装聚集体. 将制备得到的嵌段共聚物溶 解在 THF 中配成一定浓度的溶液, 然后用滤膜过滤得 到纯净的溶液. 在磁力摚拌的作用下向该溶液中以 2.5 $\mu \mathrm{L} / \mathrm{s}$ 的速度滴加蒸馏水形成聚集体.

\section{6 电镜样品制备}

将自组装形成的聚集体水溶液按照一定浓度稀释 后滴在铜网(FETEM)和硅片(SEM 和 FESEM)上, 在室 温下自然挥发溶剂. SEM 和 FESEM 的样品需要在测试 前喷金，电流 $10 \mathrm{~mA}, 110 \mathrm{~s}$.

\section{7 表征}

采用凝胶渗透色谱仪(GPC, Shimazu, LC-10ADvp) 测定大分子引发剂以及嵌段共聚物的分子量及其分布, 测试时以四氢呋喃作为流动相, 以聚苯乙烯为标样, 柱 温为 $45{ }^{\circ} \mathrm{C}$, 流速 $0.8 \mathrm{~mL} / \mathrm{min}$.

核磁共振谱 $\left({ }^{1} \mathrm{H}\right.$ NMR, Bruker, DMX500)测定时以 氝代氯仿为溶剂, 以四甲基硅烷(TMS)为内标.

采用扫描电镜(SEM, Philips, XL30FEG*)表征聚集 体形态, 电压 $20 \mathrm{kV}$.

采用场发射透射电镜(FETEM, FEI, Tecnai G2, F20) 表征聚集体形态，电压 $200 \mathrm{kV}$.

采用场发射扫描电子显微镜(FESEM, Zeiss, Ultra55)观测聚集体表面形貌，电压 $5 \mathrm{kV}$.

采用紫外-可见吸收光谱仪(Perkin Elmer, Lambda 650)测定偶氮苯基团的光致异构变化.

采用粒径分析仪(Malvern, Nano ZS90)测定聚集体 的粒径及其分布.

采用高分辨光学显微镜(HiROX, KH-7700)观察聚 集体的微观形貌.

采用荧光分光光度计(上海棱光, F97Pro)表征包覆 有荧光染料尼罗红的胶束的光响应性.

\section{References}

[1] Kazakov, S.; Kaholek, M.; Teraoka, I.; Levon, K. Macromolecules 2002, 35, 1911 .

[2] Wang, D.; Wang, X. Prog. Polym. Sci. 2013, 38, 271.

[3] Deng, Y.; Cai, Y.; Sun, Z.; Gu, D.; Wei, J.; Li, W.; Guo, X.; Yang, J.; Zhao, D. Adv. Funct. Mater. 2010, 20, 3658.

[4] Han, D.; Tong, X.; Zhao, Y. Langmuir 2012, 20, 2327.

[5] Yu, Y.; Nakano, M.; Ikeda, T. Nature 2003, 425, 145.

[6] Han, D.; Tong, X.; Zhao, Y. Macromolecules 2011, 44(3), 437.

[7] Li, N.; Ye, G.; He, Y.; Wang, X. Chem. Commun. 2011, 47(16), 4757.

[8] Li, L.; Yan, Z.; Gu, J. Macromol. Rapid Commun. 2009, 30(13), 1089.

[9] Lin, L.; Feng, Z.; Yu, Q.; Yan, Z.; Yen, C.; Yu, Y. Mol. Cryst. Liq. Cryst. 2009, 508, 579.

[10] Feng, Z.; Lin, L.; Yan, Z.; Yu, Y. Macromol. Rapid Commun. 2010, 31, 640 .

[11] Pan, G.; Feng, Z.; Wei, J.; Yu, Y. Acta Chim. Sinica 2013, 71, 733. (潘高翔, 冯泽, 韦嘉, 俞燕蕾, 化学学报, 2013, 71, 733.)

[12] Zhang, L.; Eisengberg, A. J. Am. Chem. Soc. 1996, 118, 1728.

[13] Lin, S.; Wang, Y.; Cai, C.; Xing, Y.; Lin, J.; Chen, T.; He, X. Nanotechnology 2013, 24, 1.

[14] Burke, S. E.; Eisenberg, A. Langmuir 2001, 17, 6705.

[15] Svensson, M.; Alexandridis, P.; Linse, P. Macromolecules 1999, 32, 5435 . 
[16] Feng, K.; Xie, N.; Chen, B.; Zhang, L. P.; Tung, C. H.; Wu, L. Z. Macromolecules 2012, 45, 5596.

[17] Lv, C.; Wang, Z.; Wang, P.; Tang, X. Langmuir 2012, 28, 9387.
[18] Wang, G.; Tong, X.; Zhao, Y. Macromolecules 2004, 37(24), 8911.

[19] Bai, R.; You, Y.; Pan, C. Polym. Int. 2000, 49, 898.

[20] Zhang, L.; Eisengberg, A. Science 1995, 268, 1728. 